\title{
Modulation of MicroRNAs by Phytochemicals in Cancer: Underlying Mechanisms and Translational Significance
}

\author{
Sanjeev K. Srivastava, ${ }^{1}$ Sumit Arora, ${ }^{1}$ Courey Averett, ${ }^{1}$ Seema Singh, ${ }^{1}$ and Ajay P. Singh ${ }^{1,2}$ \\ ${ }^{1}$ Mitchell Cancer Institute, University of South Alabama, Mobile, AL 36604, USA \\ ${ }^{2}$ Department of Biochemistry and Molecular Biology, University of South Alabama, Mobile, AL 36688, USA \\ Correspondence should be addressed to Ajay P. Singh; asingh@health.southalabama.edu
}

Received 26 August 2014; Accepted 12 October 2014

Academic Editor: Fumitaka Takeshita

Copyright (c) 2015 Sanjeev K. Srivastava et al. This is an open access article distributed under the Creative Commons Attribution License, which permits unrestricted use, distribution, and reproduction in any medium, provided the original work is properly cited.

MicroRNAs (miRNAs) are small, endogenous noncoding RNAs that regulate a variety of biological processes such as differentiation, development, and survival. Recent studies suggest that miRNAs are dysregulated in cancer and play critical roles in cancer initiation, progression, and chemoresistance. Therefore, exploitation of miRNAs as targets for cancer prevention and therapy could be a promising approach. Extensive evidence suggests that many naturally occurring phytochemicals regulate the expression of numerous miRNAs involved in the pathobiology of cancer. Therefore, an understanding of the regulation of miRNAs by phytochemicals in cancer, their underlying molecular mechanisms, and functional consequences on tumor pathophysiology may be useful in formulating novel strategies to combat this devastating disease. These aspects are discussed in this review paper with an objective of highlighting the significance of these observations from the translational standpoint.

\section{Introduction}

MicroRNAs (miRNAs) are endogenous, small noncoding RNA molecules that posttranscriptionally regulate gene expression. miRNAs bind to the $3^{\prime}$-UTRs of target mRNA with partial or complete complementarity, thus causing translational repression or target messenger RNA (mRNA) degradation [1]. An individual miRNA can regulate the expression of multiple genes; conversely, a single mRNA can be targeted by many miRNAs. To date, about 2,469 miRNAs have been identified in humans [2], and more than one-third of all human genes are potentially regulated by miRNAs [3]. Extensive studies have shown that miRNAs not only are involved in the process of cell development and differentiation but also play a critical role in carcinogenesis [4]. Emerging data suggest that several classes of naturally occurring, plant-derived compounds (phytochemicals) could potentially regulate the expression of several miRNAs involved in cancer.

Phytochemicals are nonnutritive plant chemicals that have various applications including anti-inflammatory and anticancer. These phytochemicals are widely distributed in various fruits, vegetables, herbs, beverages, and many other dietary supplements. Numerous studies have demonstrated that the intake of fruit- and vegetable-rich foods decreases the occurrence of cancer [5-7]. So far, more than 10,000 phytochemicals have been identified [8], and a significant number of phytochemicals show anticancer potential with no or minimal toxicity to normal cells [9]. Interestingly, around $47 \%$ of FDA approved anticancer drugs are derived from plants $[10,11]$. Moreover, these phytochemicals could be used as a single chemotherapeutic agent or in association with standard anticancer drugs. Phytochemicals can increase the efficacy of anticancer drugs synergistically, while reducing the toxic side effects of the standard chemotherapeutic drugs $[12,13]$. These phytochemicals exert their anticancer effects through modulation of multiple molecular targets affecting various signaling pathways $[8,14,15]$. In the present review paper, we focus our attention on the regulation of miRNAs by some of the phytochemicals such as resveratrol, epigallocatechin-3-gallate (EGCG), curcumin, camptothecin 
(CPT), and diindolylmethane (DIM) for cancer prevention and therapy.

\section{Biogenesis of miRNA and Mechanism of Gene Silencing}

The biosynthesis of miRNAs begins with gene transcription by RNA polymerase II or RNA polymerase III into primary miRNA (pri-miRNA) transcripts inside the nucleus. These pri-miRNAs are comprised of either a cluster or single miRNAs folded into a hairpin stem-like structure [1]. This long pri-miRNA is processed by the sequential, endonucleolytic cleavage of the transcript by the microprocessor complex, containing drosha and DGCR8, into a 65-70-nucleotide precursor miRNA (pre-miRNA). This pre-miRNA is then exported from the nucleus to the cytoplasm by the nuclear export factor Exportin-5/Ran-GTP and cleaved by RNase III endonuclease, Dicer/TRBP, and argonaute 2 (Ago2) miRNA duplex [16] into an 22 -nucleotide product. This duplex miRNA then unwinds to generate a single-stranded miRNA. One of the strands enters in the RNA-induced silencing complex (RISC) along with Ago proteins and directs the complex to target mRNA. This binding causes either target mRNA degradation or inhibition of translation [1].

\section{Biological Significance of miRNAs in Cancer: Tumor Suppressors and Oncogenes}

Based on in vitro and in vivo studies, miRNAs have been characterized as tumor suppressors or oncogenes. In this section we will discuss some of the reports from a long list of tumor suppressor/oncogenic miRNAs that have been experimentally validated.

Numerous studies have shown that the expression of the tumor suppressor Let-7 is significantly downregulated or lost in various cancers [17-19]. It has been shown that restoration of Let-7 inhibited tumor growth in a K-RAS lung cancer model [20]. Furthermore, decreased expression of let-7 correlated with shorter survival in non-small-cell lung cancer [21]. Other miRNAs such as miR-15a and miR-16 are either deleted or downregulated in most cases of the chronic lymphocytic leukemia, and their overexpression induced apoptosis [22]. Moreover, it has been reported that miR-16 and miR-15 were downregulated in multidrug-resistant human gastric cancer cells, and restoration of these miRNAs sensitized the cancer cells to chemotherapeutic drugs [23]. Takeshita and coworkers demonstrated the growth inhibitory role of miR16 in prostate cancer cells [24]. The levels of miR-34 are significantly decreased in various cancers, and its restoration was reported to inhibit angiogenesis and malignant behavior, yet at the same time it induced apoptosis and cell cycle arrest $[25,26]$. Additionally, the role of miR-34 in the inhibition of tumor-initiating cells has also been suggested [27]. In mixed lineage leukemia, the overexpression of miR-495 inhibited cell viability and reduced leukemogenesis in vivo [28]. In one of our recent studies, we investigated the significance of miR-150 downregulation in pancreatic cancer [29]. We demonstrated that the restoration of miR-150 inhibited the
MUC4 oncoprotein. Consequently, the growth and malignant potential of pancreatic cancer cells were suppressed $[29,30]$. The role of miR-451 as a tumor suppressor miRNA by inhibiting cancer cell migration, invasion, and growth is well known [31-33]. Further, Kovalchuk and coworkers demonstrated that miR-451 sensitizes MCF-7/DOX-resistant cells to doxorubicin cytotoxicity [34]. $\mathrm{Xu}$ and coworkers demonstrated that miR-203 overexpression effectively inhibited cell proliferation and induced apoptosis and cell cycle arrest. Moreover, its overexpression also inhibited tumor growth in a mouse model [35].

To date, several miRNAs have been identified that act as oncogenes [36]. miR-17-92 is an oncogenic gene cluster [37]; restoration of this cluster in MYC-driven B-cell lymphomas suppressed apoptosis and enhanced tumorigenicity [37]. Ma et al., 2010, demonstrated that miR-27a, which is overexpressed in pancreatic cancer, plays an oncogenic role by targeting tumor suppressor Spry2 [38]. Inhibition of miR-27a decreased growth, clonogenicity, and migration of pancreatic cancer cells. miR-21 is a widely studied oncogenic miRNA which is frequently overexpressed in various malignancies $[39,40]$. Inactivation of miR-21 results in apoptosis induction, inhibition of growth, and malignant progression [39-41]. In a transgenic mice model, miR-155 induced polyclonal pre-Bcell proliferation resulting in B-cell leukemia [42]. miR-373 and miR-520c are known to promote migration, invasion, and metastasis of breast cancer cells by targeting CD44 expression [43]. Moreover, the roles of miR-373 and miR$520 \mathrm{c}$ in promoting migration and invasion of prostate cancer cells have also been reported [44]. In pancreatic cancer, miR-424-5p is overexpressed, and its high expression has been reported to be associated with enhanced proliferation and apoptosis resistance through downregulating SOCS6 [45]. miR-10b is overexpressed in various malignancies and promotes cell migration, invasion, and metastasis [46-48]. Moreover, a high expression of miR-10b correlated with disease progression $[47,49]$. miR-221 and miR-222 are known to facilitate tumor cell growth, malignant potential, and EMT in multiple malignancies such as prostate, breast, and thyroid cancer [50-53].

Moreover, overexpression of miR-221 and miR-222 was shown to impart tamoxifen-resistance in breast cancer cells [54]. Altogether, these findings indicate that miRNAs play important roles as oncogenes in cancer cells.

\section{Modulation of miRNAs by Phytochemicals}

miRNAs are being considered as attractive targets for cancer prevention and therapy due to their oncogenic or tumor suppressor activities. Various studies have suggested that the modulation of miRNAs serves as one of the key mechanisms in the anticancer activities of a variety of phytochemicals (Table 1). Below, we describe some of the phytochemicals which are known to regulate miRNA expression in cancer.

4.1. Resveratrol. Resveratrol is a stilbenoid that has been shown to have anticancer activities against various cancers 
TABLE 1: Phytochemical-mediated regulation of miRNAs in cancer. Modulation of miRNA expression by certain phytochemical agents and effect on cancer pathobiology.

\begin{tabular}{|c|c|c|c|}
\hline Phytochemical & miRNA & Function & Reference \\
\hline Resveratrol & $\begin{array}{l}\uparrow \quad \text { miR-141, miR-663, and miR-200c } \\
\downarrow \text { miR-17, miR-21, miR-25, miR-92a-2, miR-103-1, and } \\
\text { miR-103-2 }\end{array}$ & Invasiveness, EMT, and metastasis & {$[57-61,114]$} \\
\hline \multirow[b]{2}{*}{ EGCG } & $\uparrow \quad \mathrm{miR}-16, \mathrm{miR}-210$, miR-7-1, miR-34a, and miR-99a & Apoptosis & \multirow{2}{*}[62-66]{} \\
\hline & $\downarrow \quad$ miR-21, miR-98-5p, miR-92, miR-93, and miR-106b & $\begin{array}{l}\text { Proliferation, anchorage-independent } \\
\text { growth, and drug resistance }\end{array}$ & \\
\hline \multirow{2}{*}{ Genistein } & $\uparrow \quad \mathrm{miR}-16$ & Apoptosis & \multirow{2}{*}[67,68]{} \\
\hline & $\downarrow \quad \mathrm{miR}-221$ and $\mathrm{miR}-222$ & Growth & \\
\hline \multirow{2}{*}{ Curcumin } & $\uparrow \quad \mathrm{miR}-15 \mathrm{a}, \mathrm{miR}-16$, and miR-186 ${ }^{*}$ & Apoptosis & \multirow{2}{*}[80,82,83]{} \\
\hline & $\downarrow \quad \mathrm{miR}-21$ & Metastasis & \\
\hline \multirow{2}{*}{ Quercetin } & $\uparrow$ let-7 & Growth & \multirow{2}{*}[88,89]{} \\
\hline & $\downarrow \quad \mathrm{miR}-27 \mathrm{a}$ & Apoptosis & \\
\hline Camptothecin & $\downarrow \quad \mathrm{miR}-125 \mathrm{~b}$ & Apoptosis & [92] \\
\hline DIM & $\begin{array}{l}\uparrow \operatorname{miR}-21, \mathrm{miR}-200 \mathrm{~b}, \mathrm{miR}-200 \mathrm{c} \text {, let-7b, let-7c, let-7d, let-7e, } \\
\text { and miR-146a }\end{array}$ & $\begin{array}{l}\text { Growth, EMT, drug resistance, invasion, } \\
\text { and metastasis }\end{array}$ & [95-97] \\
\hline
\end{tabular}

including breast cancer, lung cancer, glioma, prostate cancer, colon cancer, and neuroblastoma $[55,56]$. Resveratrol reduced the expression of numerous oncogenic miRNAs, namely, miR-17, miR-21, miR-25, miR-92a-2, miR-103-1, and miR-103-2, in human colon cancer cells [57]. Moreover, in the same study, tumor suppressor miR-663 levels were shown to be restored in human colon cancer cells after the treatment of resveratrol. In another study, Hagiwara et al. reported that resveratrol treatment upregulated miR-141 and resulted in a significant reduction of invasiveness, whereas resveratrol-induced miR-200c expression caused reversal of EMT through downregulation of Zeb1 and upregulation of E-cadherin [58]. It has been demonstrated that the anticancer effect of resveratrol on pancreatic cancer cells was due to inhibition of oncogenic miR-21 [59]. Moreover, the synergistic antitumor activity of resveratrol and miR-200c has been demonstrated in human lung cancer cells [60]. In colon cancer cells, resveratrol inhibited the cell growth and induced apoptosis through upregulating miR-34a expression [61].

4.2. Epigallocatechin-3-gallate (EGCG). Epigallocatechin-3gallate is a polyphenol flavonoid that possesses significant antioxidant and anticancer properties. It has been shown that EGCG induces apoptosis in hepatocellular carcinoma through enhanced expression of miR-16 [62]. Increased expression of miR-16 resulted in inhibition of its target antiapoptotic Bcl-2, followed by mitochondrial dysfunction, cytochrome c release, and subsequent apoptosis. EGCG also inhibited the expression of miR-21 followed by repression of androgen receptor (AR) signaling and, consequently, a reduction of prostate cancer cell growth [63]. In lung cancer, EGCG upregulated the expression of miR-210, which led to the inhibition of proliferation and anchorage-independent growth [64]. EGCG enhanced the efficacy of cisplatin through downregulation of miR-98-5p in A549 non-small lung cancer cells [65]. A combination of N-(4-hydroxyphenyl) retinamide and
EGCG decreased the expression of oncogenic miRs (miR92, miR-93, and miR-106b) and enhanced the expression of tumor suppressor miRs (miR-7-1, miR-34a, and miR99a) which resulted in growth inhibitory effects in human malignant neuroblastoma cells [66].

4.3. Genistein. Genistein is another important polyphenol that showed significant anticancer effects through the regulation of miRNAs. Genistein treatment was shown to enhance apoptosis synergistically with miR-16 in human chronic lymphocytic leukemia cells [67]. In a study on prostate cancer, genistein both downregulated miR-221 and miR-222 and restored tumor suppressor gene aplasia Ras homolog member I (ARHI) expression, which ultimately resulted in anticancer effects [68]. In another study on prostate cancer, genistein inhibited the migration and invasion of PC3 and DU145 cells through downregulating oncogenic miR-151 [69]. Xu et al. have shown that treatment of ovarian cancer cells with genistein caused an inhibition of cell growth and migration through suppression of miR-27a [70]. Further, genistein has been shown to upregulate the tumor suppressor miR574-3p in prostate cancer cells [71]. Moreover, it has been observed that genistein exerted its antitumor effect in prostate cancer via downregulation of miR-1260b [72]. Genistein treatment downregulated oncogenic miR-1260b and resulted in inhibition of Wnt-signalling in renal cancer cells [72]. miR223 expression was found to be downregulated in pancreatic cancer cells after genistein treatment that correlated with cell growth inhibition and induction of apoptosis [73]. Genistein also plays a tumor suppressor role through inhibition of miR27a in pancreatic cancer cells [74].

4.4. Curcumin. Curcumin is a constituent of turmeric (Curcuma longa) and has been used as an important component of spice in Indian food and as a traditional medicine in Asian 
countries for many decades [75]. It possesses chemopreventive and chemotherapeutic activities against many tumors [75-78]. Curcumin exerts its therapeutic effects by regulating miRNAs known to play an important role in cancer [79]. Yang et al. have shown that curcumin upregulated miR$15 \mathrm{a}$ and miR-16 in MCF-7 breast cancer cells which caused an induction of apoptosis [80]. In another study, curcumin treatment resulted in the upregulation of tumor suppressor miR-203 in bladder cancer that led to apoptosis induction and diminished proliferation, migration, and invasion [81]. Curcumin has also been shown to induce tumor suppressor miR$186 *$ expression to promote apoptosis in lung cancer [82]. Moreover, curcumin inhibited the transcriptional regulation of oncogenic miR-21 in colon cancer, causing inhibition of growth, invasion, and metastasis [83]. Zhao et al. provided evidence that curcumin exerts its cytotoxic effects against SKOV3 ovarian cancer cells largely through upregulation of miR-9 [84]. Another tumor suppressor, miR181b, has been demonstrated to be induced by curcumin, and it inhibited breast cancer metastasis via downregulation of the inflammatory cytokines CXCL1 and CXCL2 [85]. High levels of miR-221 expression have been correlated with shorter survival in pancreatic cancer patients, suggesting that miR221 could be an oncogenic miRNA [86]. In the same study, the synthetic curcumin analogue (CDF) has been found to suppress the expression of miR-221 and upregulate the expression of PTEN, p27 (kip1), p57 (kip2), and PUMA, followed by inhibition of cell proliferation and migration of pancreatic cancer cells. Thus, altogether, these studies provide evidence that curcumin modulates the expression of miRNA signatures in cancer cells to confer its anticancer activity.

4.5. Quercetin. Intake of a quercetin-rich diet has been demonstrated to modulate the expression of 48 unique miRNAs. These miRNAs have been reported to decrease tumor metastasis and invasion (miR-146a/b, 503, and 194), inhibit cell proliferation (miR-125a, 155, let-7 family, 302c, 195, 26a, 503, and 215), induce apoptosis (miR-125a, 605, 26b, let-7g, 34a, 491, and 16), and upregulate tumor suppressor miRNAs (let-7 family, miR-125a, 183, 146a, 98, 19b, 106a, and 381) [87]. Del Follo-Martinez et al. reported that quercetin treatment induced apoptosis in colorectal cancer cells when used along with resveratrol. The underlying mechanism of apoptosis induction is the downregulation of oncogenic miR-27a [88]. In another study, quercetin, when used with catechins, was shown to enhance the expression of let-7 in pancreatic cancer cells followed by K-ras inhibition and reduction of the advancement of pancreatic cancer [89].

4.6. Camptothecin (CPT). Camptothecin, an alkaloid isolated from bark of Camptotheca acuminata, is a potent chemotherapeutic agent against a variety of tumors [90-92]. CPT was demonstrated to reduce the expression of miR125b significantly, which led to the upregulation of Bak1 and p53 and resulted in apoptosis of human cervical cancer and myelogenous leukemia cells [92]. In a recent study, camptothecin was shown to inhibit HIF- $1 \alpha$ by enhancing the levels of miR-155, miR-17-5p, and miR-18a in HeLa cells [93].
4.7. Diindolylmethane (DIM). Diindolylmethane is an active compound that is generated in the stomach through the metabolic conversion of indole-3-carbinol (I3C), present in cruciferous vegetables [94]. DIM regulates the expression of numerous miRNAs involved in cancer development and progression. DIM has been demonstrated to induce the expression of certain miRNAs, such as miR-200 and let7 families, that led to the reversal of EMT and enhanced chemosensitivity in gemcitabine-resistant pancreatic cancer cells [95]. DIM also induced the expression of miR-146a, which resulted in reduced pancreatic cancer cell invasion via inhibition of metastasis-associated protein 2 (MTA2 ), interleukin-1 receptor-associated kinase 1 (IRAK-1), and $\mathrm{NF} \kappa \mathrm{B}$ [96]. Moreover, Jin observed that DIM inhibited breast cancer cell growth by enhancing the expression of miR-21 which led to the degradation of its target Cdc25A [97]. Formulated 3,3'-diindolylmethane (BR-DIM) has been shown to be capable of downregulating miR-221, resulting in growth inhibition of pancreatic cancer cells [86].

\section{Mechanism of miRNA Regulation by Phytochemicals}

There have been several aberrantly expressed miRNAs identified in various cancer types. Unfortunately, the precise mechanisms that regulate the normal expression of miRNAs or their deregulation in cancer remain unclear. Growing evidence suggests that aberrant transcriptional regulation, epigenetic changes, alterations in miRNA biosynthesis machinery, mutations, or DNA copy number could contribute to miRNA dysregulation in human cancer [98]. Experimental studies on phytochemicals revealed that the expression of various miRNAs can be regulated by phytochemicals. In the following section, we provide an overview for the regulation of miRNAs by phytochemicals through various mechanisms such as epigenetic, transcriptional, and miRNA processing (Figure 1).

5.1. Transcriptional Regulation of miRNAs. Various studies indicate the involvement of certain transcription factors in the regulation of miRNA expression and the subsequent modulation of pathological conditions in cancer. Thus, transcription factor-mediated miRNA regulation is one critical aspect of study. Several groups have observed that phytochemicals modulate miRNA expression through transcriptional regulation (Figure 1). Wang and coworkers demonstrated that treatment with EGCG results in the transcriptional activation of miR-210 in lung cancer by promoting the binding of hypoxia inducible factor- $1 \alpha$ (HIF- $1 \alpha)$ to the hypoxia response element present in the promoter region of miR-210 [64]. Transcription factor activator protein (AP-1), an important regulator of genes involved in cell proliferation and extracellular matrix production [99], is an upstream regulator of miR-21 [100]. Mudduluru and coworkers demonstrated that curcumin inhibits the transcriptional regulation of miR-21 by downregulating AP-1 to suppress tumor growth, invasion, and metastasis of colorectal cancer [83]. Another transcription factor, CCAAT/enhancer binding protein beta 


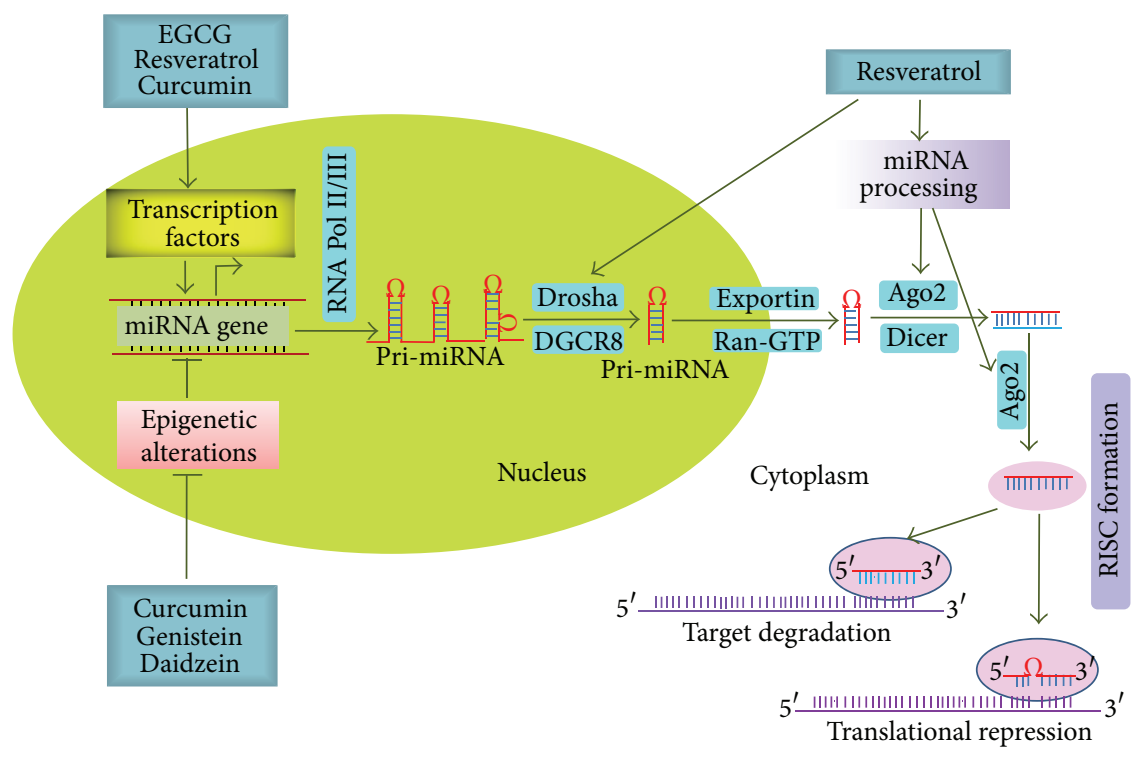

FIGURE 1: Mechanistic overview of miRNA regulation by phytochemicals. Phytochemicals regulate the expression of miRNAs through modulation of transcription factors and inducing epigenetic modifications and by interfering with processes associated with miRNA maturation.

$(\mathrm{C} / \mathrm{EBP}-\beta)$, negatively regulates tumor suppressor miR-145 by directly interacting through the putative C/EBP- $\beta$ binding site present in the miR-145 promoter. It was also shown that resveratrol treatment resulted in the decreased activation of C/EBP- $\beta$ which subsequently induced the expression of miR-145 in breast cancer cells [101]. Androgen receptor (AR) directly regulates miR-21 by binding to the miR-21 promoter in prostate cancer [102]; Siddiqui and coworkers (2011) demonstrated that EGCG inhibited prostate cancer cell growth by decreasing the level of AR and miR-21 [63]. A study conducted by Hagiwara and coworkers has shown that resveratrol transcriptionally upregulated the expression of several tumor suppressor miRNAs such as miR-141, miR-26a, miR-195, miR-126, miR-340, miR-34a, miR-193b, miR-335, miR-200c, miR-497, miR-196a, and miR-125a-3p in MDAMB-231 breast cancer cells [58]. It is known that p53 is a tumor suppressor gene and a transcription factor which functions by either causing growth arrest or inducing apoptosis [103]. Emerging data suggest that p53 transcriptionally regulates several miRNAs [104]. A number of studies have shown that curcumin regulates the expression of several miRNAs that are transcriptional targets of p53 such as miR-22, miR-15/16a, miR-34, and miR-21 [80, 83, 105-108]. Considering all these observations, it is speculated that curcumin regulates the expression of $\mathrm{p} 53$, which in turn modulates the expression of several miRNAs.

5.2. Epigenetic Regulation of miRNAs. Epigenetic regulation is defined as modifications of the genome without any change to the nucleotide sequence. Supporting evidence suggests that epigenetic modification, like aberrant $\mathrm{CpG}$ methylation or histone modifications, contributes to the dysregulation of gene expression in tumor cells. Pharmacologic inhibition of
DNA methyltransferase (DNMT) causes DNA demethylation and upregulates the expression of miRNAs. Saini and coworkers provided evidence that curcumin treatment resulted in the hypomethylation of miR-203 promoter and subsequent upregulation of miR-203, which is epigenetically silenced in various malignancies [81]. Treatment with a curcumin analogue, difluorinated curcumin, restored the expression of miR-34a and miR-34c by working as a demethylating agent in colon cancer cells [109]. Rabiau and coworkers performed miRNA expression profiling following the treatment with the flavonoids genistein and daidzein in prostate cancer cells [110]. Their investigation revealed a significant upregulation of miR-548b-5p in PC3 and miR-15a in LNCaP cells by genistein and daidzein through regulation at the epigenetic level [110]. Zaman et al. demonstrated that miR-145 is inactivated as a result of its promoter methylation in prostate cancer; treatment with genistein demethylated the promoter of miR145 which resulted in an increased level of miR-145 [111]. In a separate study on prostate cancer, isoflavone efficiently demethylated the promoter region of miR-29a and miR-1256 and subsequently upregulated their expression [112]. All these observations suggest the significant role of phytochemicals in the regulation of miRNAs at the epigenetic level (Figure 1).

5.3. Regulation of miRNA Processing by Phytochemicals. miRNA processing can be regulated at various steps, and any alteration in processing either increases or decreases the level of miRNAs [113]. The role of phytochemical-modulated expression of miRNAs through the regulation of proteins involved in miRNA processing has been recently investigated, as shown in Figure 1. Hagiwara and coworkers investigated and reported that resveratrol treatment significantly increased the expression of Ago2 and results in enhanced 
levels of tumor suppressor miRNAs such as miR-16, miR141, miR-143, and miR-200c in MDA-MB-231 cells [58]. Moreover, resveratrol treatment resulted in the enhanced level of miR-663 and pre-miR- 663 by interfering with the drosha mediated processing of pri-miR-663 that resulted in the inhibition of miR-155, which is overexpressed in many cancers [114]. These studies provide evidence that phytochemicals regulate the expression of miRNAs epigenetically and transcriptionally and by controlling miRNA processing.

\section{Conclusion and Future Perspective}

miRNAs are a novel class of gene regulators. Thus far, we have only been able to see just a small part of the complexity of cellular regulation by miRNAs with regard to all of the different functions that miRNAs can perform. miRNAs are aberrantly expressed in most cancers and this has been correlated throughout cancer initiation and progression; hence, miRNAs represent very attractive and novel targets for cancer therapy. Phytochemicals display an inimitable ability to alter the level of miRNAs involved in regulation of cancer pathobiology by modulating the expression of miRNAs through a variety of mechanisms, namely, epigenetic, transcriptional, and miRNA processing. In addition, phytochemicals increase the chemosensitivity of conventional therapeutic drugs through modulating miRNAs. As a result, phytochemicals can be exploited for designing therapeutic approaches in combination with conventional therapies to improve cancer treatment and prevention strategies. Despite the potent anticancer activities of phytochemicals, there are some concerns such as specific targeting and bioavailability. To overcome these hurdles in the use of phytochemicals, different approaches are being explored, namely, chemical modification, synthetic formulation, delivery by nanoparticles, and so forth. Hence, there is a wide scope for the development of phytochemicals into commercial drugs to efficaciously prevent and treat cancer.

\section{Conflict of Interests}

The authors declare that there is no conflict of interests regarding the publication of this paper.

\section{Acknowledgments}

Ajay P. Singh received Grants nos. CA137513, CA167137, CA175772, and CA185490 and Seema Singh received Grant no. CA169829 by NIH/NCI. Ajay P. Singh received Grants nos. PC110545 and PC0739930 by DOD/US Army, and internal funding support from USAMCI.

\section{References}

[1] A. Bhardwaj, S. Singh, and A. P. Singh, "MicroRNA-based cancer therapeutics: big hope from small RNAs," Molecular and Cellular Pharmacology, vol. 2, no. 5, pp. 213-219, 2010.

[2] M. R. Friedländer, E. Lizano, A. J. S. Houben et al., "Evidence for the biogenesis of more than 1,000 novel human microRNAs," Genome Biology, vol. 15, no. 4, article R57, 2014.
[3] S. Srivastava, A. Bhardwaj, S. Leavesley, W. Grizzle, S. Singh, and A. Singh, "MicroRNAs as potential clinical biomarkers: emerging approaches for their detection," Biotechnic \& Histochemistry, vol. 88, no. 7, pp. 373-387, 2013.

[4] S. K. Srivastava, S. Arora, S. Singh, A. Bhardwaj, C. Averett, and A. P. Singh, "MicroRNAs in pancreatic malignancy: progress and promises," Cancer Letters, vol. 347, no. 2, pp. 167-174, 2014.

[5] G. Block, B. Patterson, and A. Subar, "Fruit, vegetables, and cancer prevention: a review of the epidemiological evidence," Nutrition and Cancer, vol. 18, no. 1, pp. 1-29, 1992.

[6] L. Reddy, B. Odhav, and K. D. Bhoola, "Natural products for cancer prevention: a global perspective," Pharmacology and Therapeutics, vol. 99, no. 1, pp. 1-13, 2003.

[7] K. A. Steinmetz and J. D. Potter, "Vegetables, fruit, and cancer prevention: a review," Journal of the American Dietetic Association, vol. 96, no. 10, pp. 1027-1039, 1996.

[8] M. Russo, C. Spagnuolo, I. Tedesco, and G. L. Russo, "Phytochemicals in cancer prevention and therapy: truth or dare?" Toxins, vol. 2, no. 4, pp. 517-551, 2010.

[9] C. V. Rao, C.-X. Wang, B. Simi et al., "Enhancement of experimental colon cancer by genistein," Cancer Research, vol. 57, no. 17, pp. 3717-3722, 1997.

[10] D. J. Newman and G. M. Cragg, "Natural products as sources of new drugs over the last 25 years," Journal of Natural Products, vol. 70, no. 3, pp. 461-477, 2007.

[11] G. L. Russo, "Ins and outs of dietary phytochemicals in cancer chemoprevention," Biochemical Pharmacology, vol. 74, no. 4, pp. 533-544, 2007.

[12] Y. H. Siddique, G. Ara, T. Beg, J. Gupta, and M. Afzal, "Assessment of cell viability, lipid peroxidation and quantification of DNA fragmentation after the treatment of anticancerous drug mitomycin $\mathrm{C}$ and curcumin in cultured human blood lymphocytes," Experimental and Toxicologic Pathology, vol. 62, no. 5, pp. 503-508, 2010.

[13] W. Tan, J. Lu, M. Huang et al., "Anti-cancer natural products isolated from chinese medicinal herbs," Chinese Medicine, vol. 6, article 27, 2011.

[14] N. Tyagi and P. C. Ghosh, "Folate receptor mediated targeted delivery of ricin entrapped into sterically stabilized liposomes to human epidermoid carcinoma (KB) cells: effect of monensin intercalated into folate-tagged liposomes," European Journal of Pharmaceutical Sciences, vol. 43, no. 4, pp. 343-353, 2011.

[15] N. Tyagi, S. S. Rathore, and P. C. Ghosh, "Enhanced killing of human epidermoid carcinoma (KB) cells by treatment with ricin encapsulated into sterically stabilized liposomes in combination with monensin," Drug Delivery, vol. 18, no. 6, pp. 394-404, 2011.

[16] J. Winter, S. Jung, S. Keller, R. I. Gregory, and S. Diederichs, "Many roads to maturity: microRNA biogenesis pathways and their regulation," Nature Cell Biology, vol. 11, no. 3, pp. 228-234, 2009.

[17] S. M. Johnson, H. Grosshans, J. Shingara et al., "RAS is regulated by the let-7 microRNA family," Cell, vol. 120, no. 5, pp. 635-647, 2005.

[18] F. Yu, H. Yao, P. Zhu et al., "let-7 regulates self renewal and tumorigenicity of breast cancer cells," Cell, vol. 131, no. 6, pp. 1109-1123, 2007.

[19] B. Zhang, X. Pan, G. P. Cobb, and T. A. Anderson, "microRNAs as oncogenes and tumor suppressors," Developmental Biology, vol. 302, no. 1, pp. 1-12, 2007. 
[20] M. S. Kumar, S. J. Erkeland, R. E. Pester et al., "Suppression of non-small cell lung tumor development by the let-7 microRNA family," Proceedings of the National Academy of Sciences of the United States of America, vol. 105, no. 10, pp. 3903-3908, 2008.

[21] X.-M. Xia, W.-Y. Jin, R.-Z. Shi, Y.-F. Zhang, and J. Chen, "Clinical significance and the correlation of expression between let-7 and K-ras in non-small cell lung cancer," Oncology Letters, vol. 1, no. 6, pp. 1045-1047, 2010.

[22] A. Cimmino, G. A. Calin, M. Fabbri et al., "miR-15 and miR-16 induce apoptosis by targeting BCL2," Proceedings of the National Academy of Sciences of the United States of America, vol. 102, no. 39, pp. 13944-13949, 2005.

[23] L. Xia, D. Zhang, R. Du et al., "miR-15b and miR-16 modulate multidrug resistance by targeting BCL2 in human gastric cancer cells," International Journal of Cancer, vol. 123, no. 2, pp. 372379, 2008.

[24] F. Takeshita, L. Patrawala, M. Osaki et al., "Systemic delivery of synthetic microRNA-16 inhibits the growth of metastatic prostate tumors via downregulation of multiple cell-cycle genes," Molecular Therapy, vol. 18, no. 1, pp. 181-187, 2010.

[25] T.-C. Chang, E. A. Wentzel, O. A. Kent et al., "Transactivation of miR-34a by p53 broadly influences gene expression and promotes apoptosis," Molecular Cell, vol. 26, no. 5, pp. 745-752, 2007.

[26] A. Javeri, M. Ghaffarpour, M. F. Taha, and M. Houshmand, "Downregulation of miR-34a in breast tumors is not associated with either p53 mutations or promoter hypermethylation while it correlates with metastasis," Medical Oncology, vol. 30, no. 1, article 413, 2013.

[27] Q. Ji, X. Hao, M. Zhang et al., "MicroRNA miR-34 inhibits human pancreatic cancer tumor-initiating cells," PLOS ONE, vol. 4, no. 8, Article ID e6816, 2009.

[28] X. Jiang, H. Huang, Z. Li et al., "miR-495 is a tumor-suppressor microRNA down-regulated in MLL-rearranged leukemia," Proceedings of the National Academy of Sciences of the United States of America, vol. 109, no. 47, pp. 19397-19402, 2012.

[29] S. K. Srivastava, A. Bhardwaj, S. Singh et al., "MicroRNA-150 directly targets MUC4 and suppresses growth and malignant behavior of pancreatic cancer cells," Carcinogenesis, vol. 32, no. 12, pp. 1832-1839, 2011.

[30] S. Arora, S. K. Swaminathan, A. Kirtane et al., "Synthesis, characterization, and evaluation of poly (D,L-lactide-coglycolide)-based nanoformulation of miRNA-150: potential implications for pancreatic cancer therapy," International Journal of Nanomedicine, vol. 9, pp. 2933-2942, 2014.

[31] D. Liu, C. Liu, X. Wang, S. Ingvarsson, and H. Chen, "MicroRNA-451 suppresses tumor cell growth by downregulating IL6R gene expression," Cancer Epidemiology, vol. 38, no. 1, pp. 85-92, 2014.

[32] X. Liu, X. Zhang, J. Xiang, Y. Lv, and J. Shi, "miR-451: potential role as tumor suppressor of human hepatoma cell growth and invasion," International Journal of Oncology, vol. 45, pp. 739745, 2014.

[33] G. Lv, Z. Hu, Y. Tie et al., "MicroRNA-451 regulates activating transcription factor 2 expression and inhibits liver cancer cell migration," Oncology Reports, vol. 32, no. 3, pp. 1021-1028, 2014.

[34] O. Kovalchuk, J. Filkowski, J. Meservy et al., "Involvement of microRNA-451 in resistance of the MCF-7 breast cancer cells to chemotherapeutic drug doxorubicin," Molecular Cancer Therapeutics, vol. 7, no. 7, pp. 2152-2159, 2008.

[35] D. Xu, Q. Wang, Y. An, and L. Xu, "MiR-203 regulates the proliferation, apoptosis and cell cycle progression of pancreatic cancer cells by targeting Survivin," Molecular Medicine Reports, vol. 8, no. 2, pp. 379-384, 2013.

[36] L. Zhang and G. Coukos, "MicroRNAs: a new insight into cancer genome," Cell Cycle, vol. 5, no. 19, pp. 2216-2219, 2006.

[37] L. He, J. M. Thomson, M. T. Hemann et al., "A microRNA polycistron as a potential human oncogene," Nature, vol. 435, no. 7043, pp. 828-833, 2005.

[38] Y. Ma, S. Yu, W. Zhao, Z. Lu, and J. Chen, "miR-27a regulates the growth, colony formation and migration of pancreatic cancer cells by targeting Sprouty2," Cancer Letters, vol. 298, no. 2, pp. $150-158,2010$.

[39] P. P. Medina, M. Nolde, and F. J. Slack, “OncomiR addiction in an in vivo model of microRNA-21-induced pre-B-cell lymphoma," Nature, vol. 467, no. 7311, pp. 86-90, 2010.

[40] F. Sicard, M. Gayral, H. Lulka, L. Buscail, and P. Cordelier, “Targeting miR-21 for the therapy of pancreatic cancer," Molecular Therapy, vol. 21, no. 5, pp. 986-994, 2013.

[41] Y. Li, L. Yan, W. Zhang et al., "miR-21 inhibitor suppresses proliferation and migration of nasopharyngeal carcinoma cells through down-regulation of BCL2 expression," International Journal of Clinical and Experimental Pathology, vol. 7, pp. 34783487, 2014.

[42] S. Costinean, N. Zanesi, Y. Pekarsky et al., "Pre-B cell proliferation and lymphoblastic leukemia/high-grade lymphoma in $\mathrm{E} \mu$ miR155 transgenic mice," Proceedings of the National Academy of Sciences of the United States of America, vol. 103, no. 18, pp. 7024-7029, 2006.

[43] Q. Huang, K. Gumireddy, M. Schrier et al., "The microRNAs miR-373 and miR-520c promote tumour invasion and metastasis," Nature Cell Biology, vol. 10, no. 2, pp. 202-210, 2008.

[44] K. Yang, A. M. Handorean, and K. A. Iczkowski, "MicroRNAs 373 and $520 \mathrm{c}$ are downregulated in prostate cancer, suppress CD44 translation and enhance invasion of prostate cancer cells in vitro," International Journal of Clinical and Experimental Pathology, vol. 2, no. 4, pp. 361-369, 2009.

[45] K. Wu, G. Hu, X. He et al., "MicroRNA-424-5p suppresses the expression of socs6 in pancreatic cancer," Pathology \& Oncology Research, vol. 19, no. 4, pp. 739-748, 2013.

[46] Y. Liu, M. Li, G. Zhang, and Z. Pang, "MicroRNA-10b overexpression promotes non-small cell lung cancer cell proliferation and invasion," European Journal of Medical Research, vol. 18, no. 1, article 41, 2013.

[47] L. Ma, J. Teruya-Feldstein, and R. A. Weinberg, "Tumour invasion and metastasis initiated by microRNA-10b in breast cancer," Nature, vol. 449, no. 7163, pp. 682-688, 2007.

[48] H. Xiao, H. Li, G. Yu et al., "MicroRNA-10b promotes migration and invasion through KLF4 and HOXD10 in human bladder cancer," Oncology Reports, vol. 31, pp. 1832-1838, 2014.

[49] A. Ahmad, S. Sethi, W. Chen, R. Ali-Fehmi, S. Mittal, and F. H. Sarkar, "Up-regulation of microRNA-10b is associated with the development of breast cancer brain metastasis," The American Journal of Translational Research, vol. 6, pp. 384-390, 2014.

[50] M. S. Hwang, N. Yu, S. Y. Stinson et al., "miR-221/222 targets adiponectin receptor 1 to promote the epithelial-tomesenchymal transition in breast cancer," PLoS ONE, vol. 8, no. 6, Article ID e66502, 2013.

[51] S. Mardente, E. Mari, F. Consorti et al., "HMGB1 induces the overexpression of miR-222 and miR-221 and increases growth and motility in papillary thyroid cancer cells," Oncology Reports, vol. 28 , no. 6 , pp. $2285-2289,2012$. 
[52] R. Nassirpour, P. P. Mehta, S. M. Baxi, and M.-J. Yin, "miR-221 promotes tumorigenesis in human triple negative breast cancer cells," PLoS ONE, vol. 8, no. 4, Article ID e62170, 2013.

[53] X. Yang, Y. Yang, R. Gan et al., "Down-regulation of mir-221 and mir-222 restrain prostate cancer cell proliferation and migration that is partly mediated by activation of SIRT1," PLOS ONE, vol. 9, no. 6, Article ID e98833, 2014.

[54] T. E. Miller, K. Ghoshal, B. Ramaswamy et al., "MicroRNA221/222 confers tamoxifen resistance in breast cancer by targeting p27Kipl," The Journal of Biological Chemistry, vol. 283, no. 44, pp. 29897-29903, 2008.

[55] A. Bishayee, T. Politis, and A. S. Darvesh, "Resveratrol in the chemoprevention and treatment of hepatocellular carcinoma," Cancer Treatment Reviews, vol. 36, no. 1, pp. 43-53, 2010.

[56] M. Athar, J. H. Back, X. Tang et al., "Resveratrol: a review of preclinical studies for human cancer prevention," Toxicology and Applied Pharmacology, vol. 224, no. 3, pp. 274-283, 2007.

[57] E. Tili, J. J. Michaille, H. Alder et al., "Resveratrol modulates the levels of microRNAs targeting genes encoding tumorsuppressors and effectors of TGF $\beta$ signaling pathway in SW480 cells," Biochemical Pharmacology, vol. 80, no. 12, pp. 2057-2065, 2010.

[58] K. Hagiwara, N. Kosaka, Y. Yoshioka, R. U. Takahashi, F. Takeshita, and T. Ochiya, "Stilbene derivatives promote Ago2dependent tumour-suppressive microRNA activity," Scientific Reports, vol. 2, article 314, 2012.

[59] P. Liu, H. Liang, Q. Xia et al., "Resveratrol induces apoptosis of pancreatic cancers cells by inhibiting miR-21 regulation of BCL2 expression," Clinical and Translational Oncology, vol. 15, no. 9, pp. 741-746, 2013.

[60] T. Bai, D.-S. Dong, and L. Pei, "Synergistic antitumor activity of resveratrol and miR-200c in human lung cancer," Oncology Reports, vol. 31, no. 5, pp. 2293-2297, 2014.

[61] M. Kumazaki, S. Noguchi, Y. Yasui et al., "Anti-cancer effects of naturally occurring compounds through modulation of signal transduction and miRNA expression in human colon cancer cells," Journal of Nutritional Biochemistry, vol. 24, no. 11, pp. 1849-1858, 2013.

[62] W. P. Tsang and T. T. Kwok, "Epigallocatechin gallate upregulation of miR-16 and induction of apoptosis in human cancer cells," The Journal of Nutritional Biochemistry, vol. 21, no. 2, pp. 140-146, 2010.

[63] I. A. Siddiqui, M. Asim, B. B. Hafeez, V. M. Adhami, R. S. Tarapore, and H. Mukhtar, "Green tea polyphenol EGCG blunts androgen receptor function in prostate cancer," The FASEB Journal, vol. 25, no. 4, pp. 1198-1207, 2011.

[64] H. Wang, S. Bian, and C. S. Yang, "Green tea polyphenol EGCG suppresses lung cancer cell growth through upregulating miR210 expression caused by stabilizing HIF-1 $\alpha$," Carcinogenesis, vol. 32, no. 12, pp. 1881-1889, 2011.

[65] D.-H. Zhou, X. Wang, and Q. Feng, "EGCG enhances the efficacy of cisplatin by downregulating hsa-miR-98-5p in NSCLC A549 cells," Nutrition and Cancer, vol. 66, no. 4, pp. 636-644, 2014.

[66] M. Chakrabarti, W. Ai, N. L. Banik, and S. K. Ray, "Overexpression of miR-7-1 increases efficacy of green tea polyphenols for induction of apoptosis in human malignant neuroblastoma SHSY5Y and SK-N-DZ cells," Neurochemical Research, vol. 38, no. 2, pp. 420-432, 2013.

[67] E. Salerno, B. J. Scaglione, F. D. Coffman et al., "Correcting miR$15 \mathrm{a} / 16$ genetic defect in New Zealand Black mouse model of CLL enhances drug sensitivity," Molecular Cancer Therapeutics, vol. 8, no. 9, pp. 2684-2692, 2009.

[68] Y. Chen, M. S. Zaman, G. Deng et al., "MicroRNAs 221/222 and genistein-mediated regulation of ARHI tumor suppressor gene in prostate cancer," Cancer Prevention Research, vol. 4, no. 1, pp. 76-86, 2011.

[69] T. Chiyomaru, S. Yamamura, M. S. Zaman et al., "Genistein suppresses prostate cancer growth through inhibition of oncogenic microRNA-151," PLoS ONE, vol. 7, no. 8, Article ID e43812, 2012.

[70] L. Xu, J. Xiang, J. Shen et al., "Oncogenic microRNA-27a is a target for genistein in ovarian cancer cells," Anti-Cancer Agents in Medicinal Chemistry, vol. 13, no. 7, pp. 1126-1132, 2013.

[71] T. Chiyomaru, S. Yamamura, S. Fukuhara et al., "Genistein up-regulates tumor suppressor microRNA-574-3p in prostate cancer," PLoS ONE, vol. 8, no. 3, Article ID e58929, 2013.

[72] H. Hirata, K. Ueno, K. Nakajima et al., "Genistein downregulates onco-miR-1260b and inhibits Wnt-signalling in renal cancer cells," British Journal of Cancer, vol. 108, no. 10, pp. 20702078, 2013.

[73] J. Ma, L. Cheng, H. Liu et al., "Genistein down-regulates miR223 expression in pancreatic cancer cells," Current Drug Targets, vol. 14, no. 10, pp. 1150-1156, 2013.

[74] J. Xia, L. Cheng, C. Mei et al., "Genistein inhibits cell growth and invasion through regulation of MiR-27a in pancreatic cancer cells," Current Pharmaceutical Design, vol. 20, no. 33, pp. 53485353, 2014.

[75] G. N. Asher and K. Spelman, "Clinical utility of curcumin extract," Alternative Therapies in Health and Medicine, vol. 19, no. 2, pp. 20-22, 2013.

[76] T. O. Khor, Y.-S. Keum, W. Lin et al., "Combined inhibitory effects of curcumin and phenethyl isothiocyanate on the growth of human PC-3 prostate xenografts in immunodeficient mice," Cancer Research, vol. 66, no. 2, pp. 613-621, 2006.

[77] X.-D. Sun, X.-E. Liu, and D.-S. Huang, "Curcumin reverses the epithelial-mesenchymal transition of pancreatic cancer cells by inhibiting the Hedgehog signaling pathway," Oncology Reports, vol. 29, no. 6, pp. 2401-2407, 2013.

[78] H. K. Vyas, R. Pal, R. Vishwakarma, N. K. Lohiya, and G. P. Talwar, "Selective killing of leukemia and lymphoma cells ectopically expressing hCG $\beta$ by a conjugate of curcumin with an antibody against hCG $\beta$ subunit," Oncology, vol. 76, no. 2, pp. 101-111, 2009.

[79] S. Reuter, S. C. Gupta, B. Park, A. Goel, and B. B. Aggarwal, "Epigenetic changes induced by curcumin and other natural compounds," Genes and Nutrition, vol. 6, no. 2, pp. 93-108, 2011.

[80] J. Yang, Y. Cao, J. Sun, and Y. Zhang, "Curcumin reduces the expression of Bcl-2 by upregulating miR-15a and miR-16 in MCF-7 cells," Medical Oncology, vol. 27, no. 4, pp. 1114-1118, 2010.

[81] S. Saini, S. Arora, S. Majid et al., "Curcumin modulates microRNA-203-mediated regulation of the Src-Akt axis in bladder cancer," Cancer Prevention Research, vol. 4, no. 10, pp. 1698-1709, 2011.

[82] J. Zhang, Y. Du, C. Wu et al., "Curcumin promotes apoptosis in human lung adenocarcinoma cells through miR- ${ }^{186} *$ signaling pathway," Oncology Reports, vol. 24, no. 5, pp. 1217-1223, 2010.

[83] G. Mudduluru, J. N. George-William, S. Muppala et al., "Curcumin regulates miR-21 expression and inhibits invasion and metastasis in colorectal cancer," Bioscience Reports, vol. 31, no. 3, pp. 185-197, 2011. 
[84] S. F. Zhao, X. Zhang, X. J. Zhang, X. Q. Shi, Z. J. Yu, and Q. C. Kan, "Induction of microRNA-9 mediates cytotoxicity of curcumin against SKOV3 ovarian cancer cells," Asian Pacific Journal of Cancer Prevention, vol. 15, pp. 3363-3368, 2014.

[85] E. Kronski, M. E. Fiori, O. Barbieri et al., "MiR181b is induced by the chemopreventive polyphenol curcumin and inhibits breast cancer metastasis via down-regulation of the inflammatory cytokines CXCL1 and -2," Molecular Oncology, vol. 8, no. 3, pp. 581-595, 2014.

[86] S. Sarkar, H. Dubaybo, S. Ali et al., "Down-regulation of miR221 inhibits proliferation of pancreatic cancer cells through up-regulation of PTEN, p27(kip1), p57(kip2), and PUMA," American Journal of Cancer Research, vol. 3, no. 5, pp. 465-477, 2013.

[87] T. K. Lam, S. Shao, Y. Zhao et al., "Influence of quercetin-rich food intake on microRNA expression in lung cancer tissues," Cancer Epidemiology, Biomarkers \& Prevention, vol. 21, no. 12, pp. 2176-2184, 2012.

[88] A. Del Follo-Martinez, N. Banerjee, X. Li, S. Safe, and S. Mertens-Talcott, "Resveratrol and quercetin in combination have anticancer activity in colon cancer cells and repress oncogenic microRNA-27a," Nutrition and Cancer, vol. 65, no. 3, pp. 494-504, 2013.

[89] M. Appari, K. R. Babu, A. Kaczorowski, W. Gross, and I. Herr, "Sulforaphane, quercetin and catechins complement each other in elimination of advanced pancreatic cancer by miRlet-7 induction and K-ras inhibition," International Journal of Oncology, vol. 45, no. 4, pp. 1391-1400, 2014.

[90] C. G. Moertel, A. J. Schutt, R. J. Reitemeier, and R. G. Hahn, "Phase II study of camptothecin (NSC-100880) in the treatment of advanced gastrointestinal cancer," Cancer Chemotherapy Reports, vol. 56, no. 1, pp. 95-101, 1972.

[91] H. Ulukan and P. W. Swaan, "Camptothecins: a review of their chemotherapeutic potential," Drugs, vol. 62, no. 14, pp. 20392057, 2002.

[92] C.-W. Zeng, X.-J. Zhang, K.-Y. Lin et al., "Camptothecin induces apoptosis in cancer cells via microRNA-125b-mediated mitochondrial pathways," Molecular Pharmacology, vol. 81, no. 4, pp. 578-586, 2012.

[93] D. Bertozzi, J. Marinello, S. G. Manzo, F. Fornari, L. Gramantieri, and G. Capranico, "The natural inhibitor of DNA topoisomerase I, camptothecin, modulates HIF-1 $\alpha$ activity by changing miR expression patterns in human cancer cells," Molecular Cancer Therapeutics, vol. 13, no. 1, pp. 239-248, 2013.

[94] D. M. Minich and J. S. Bland, "A review of the clinical efficacy and safety of cruciferous vegetable phytochemicals," Nutrition Reviews, vol. 65, no. 6, pp. 259-267, 2007.

[95] Y. Li, T. G. Vandenboom II, D. Kong et al., "Up-regulation of miR-200 and let-7 by natural agents leads to the reversal of epithelial-to-mesenchymal transition in gemcitabine-resistant pancreatic cancer cells," Cancer Research, vol. 69, no. 16, pp. 6704-6712, 2009.

[96] Y. Li, T. G. VandenBoom, Z. Wang et al., "miR-146a suppresses invasion of pancreatic cancer cells," Cancer Research, vol. 70, no. 4, pp. 1486-1495, 2010.

[97] Y. Jin, "3,3/-Diindolylmethane inhibits breast cancer cell growth via miR-21-mediated Cdc25A degradation," Molecular and Cellular Biochemistry, vol. 358, no. 1-2, pp. 345-354, 2011.

[98] S. Deng, G. A. Calin, C. M. Croce, G. Coukos, and L. Zhang, "Mechanisms of microRNA deregulation in human cancer," Cell Cycle, vol. 7, no. 17, pp. 2643-2646, 2008.
[99] B. W. Ozanne, L. McGarry, H. J. Spence et al., “Transcriptional regulation of cell invasion: AP-1 regulation of a multigenic invasion programme," European Journal of Cancer, vol. 36, no. 13, pp. 1640-1648, 2000.

[100] A. Misawa, R. Katayama, S. Koike, A. Tomida, T. Watanabe, and N. Fujita, "AP-1-dependent miR-21 expression contributes to chemoresistance in cancer stem cell-like SP cells," Oncology Research, vol. 19, no. 1, pp. 23-33, 2010.

[101] M. Sachdeva, Q. Liu, J. Cao, Z. Lu, and Y.-Y. Mo, "Negative regulation of miR-145 by C/EBP- $\beta$ through the Akt pathway in cancer cells," Nucleic Acids Research, vol. 40, no. 14, pp. 66836692, 2012.

[102] J. Ribas, X. Ni, M. Haffner et al., "miR-21: an androgen receptor-regulated microRNA that promotes hormonedependent and hormone-independent prostate cancer growth," Cancer Research, vol. 69, no. 18, pp. 7165-7169, 2009.

[103] L. Boominathan, “The tumor suppressors p53, p63, and p73 are regulators of microRNA processing complex," PLOS ONE, vol. 5, no. 5, Article ID e10615, 2010.

[104] M. Yamakuchi, C. D. Lotterman, C. Bao et al., "P53-induced microRNA-107 inhibits HIF-1 and tumor angiogenesis," Proceedings of the National Academy of Sciences of the United States of America, vol. 107, no. 14, pp. 6334-6339, 2010.

[105] X. Ma, S. N. Choudhury, X. Hua, Z. Dai, and Y. Li, "Interaction of the oncogenic miR-21 microRNA and the p53 tumor suppressor pathway," Carcinogenesis, vol. 34, no. 6, pp. 1216-1223, 2013.

[106] S. Sreenivasan, K. Thirumalai, R. Danda, and S. Krishnakumar, "Effect of curcumin on miRNA expression in human Y79 retinoblastoma cells," Current Eye Research, vol. 37, no. 5, pp. 421-428, 2012.

[107] D. Subramaniam, S. Ponnurangam, P. Ramamoorthy et al., "Curcumin induces cell death in esophageal cancer cells through modulating Notch signaling," PLOS ONE, vol. 7, no. 2, Article ID e30590, 2012.

[108] M. Yamakuchi and C. J. Lowenstein, "MiR-34, SIRT1 and p53: the feedback loop," Cell Cycle, vol. 8, no. 5, pp. 712-715, 2009.

[109] S. Roy, E. Levi, A. P. Majumdar, and F. H. Sarkar, "Expression of miR-34 is lost in colon cancer which can be re-expressed by a novel agent CDF," Journal of Hematology \& Oncology, vol. 5, article 58, 2012.

[110] N. Rabiau, H.-K. Trraf, M. Adjakly et al., "miRNAs differentially expressed in prostate cancer cell lines after soy treatment," In Vivo, vol. 25, no. 6, pp. 917-921, 2011.

[111] M. S. Zaman, Y. Chen, G. Deng et al., "The functional significance of microRNA-145 in prostate cancer," British Journal of Cancer, vol. 103, no. 2, pp. 256-264, 2010.

[112] Y. Li, D. Kong, A. Ahmad, B. Bao, G. Dyson, and F. H. Sarkar, "Epigenetic deregulation of miR-29a and miR-1256 by isoflavone contributes to the inhibition of prostate cancer cell growth and invasion," Epigenetics, vol. 7, no. 8, pp. 940-949, 2012.

[113] I. Slezak-Prochazka, D. Selvi, B.-J. Kroesen, and A. Van Den Berg, "MicroRNAs, macrocontrol: regulation of miRNA processing," RNA, vol. 16, no. 6, pp. 1087-1095, 2010.

[114] E. Tili, J.-J. Michaille, B. Adair et al., "Resveratrol decreases the levels of miR-155 by upregulating miR-663, a microRNA targeting JunB and JunD," Carcinogenesis, vol. 31, no. 9, pp. 15611566, 2010. 

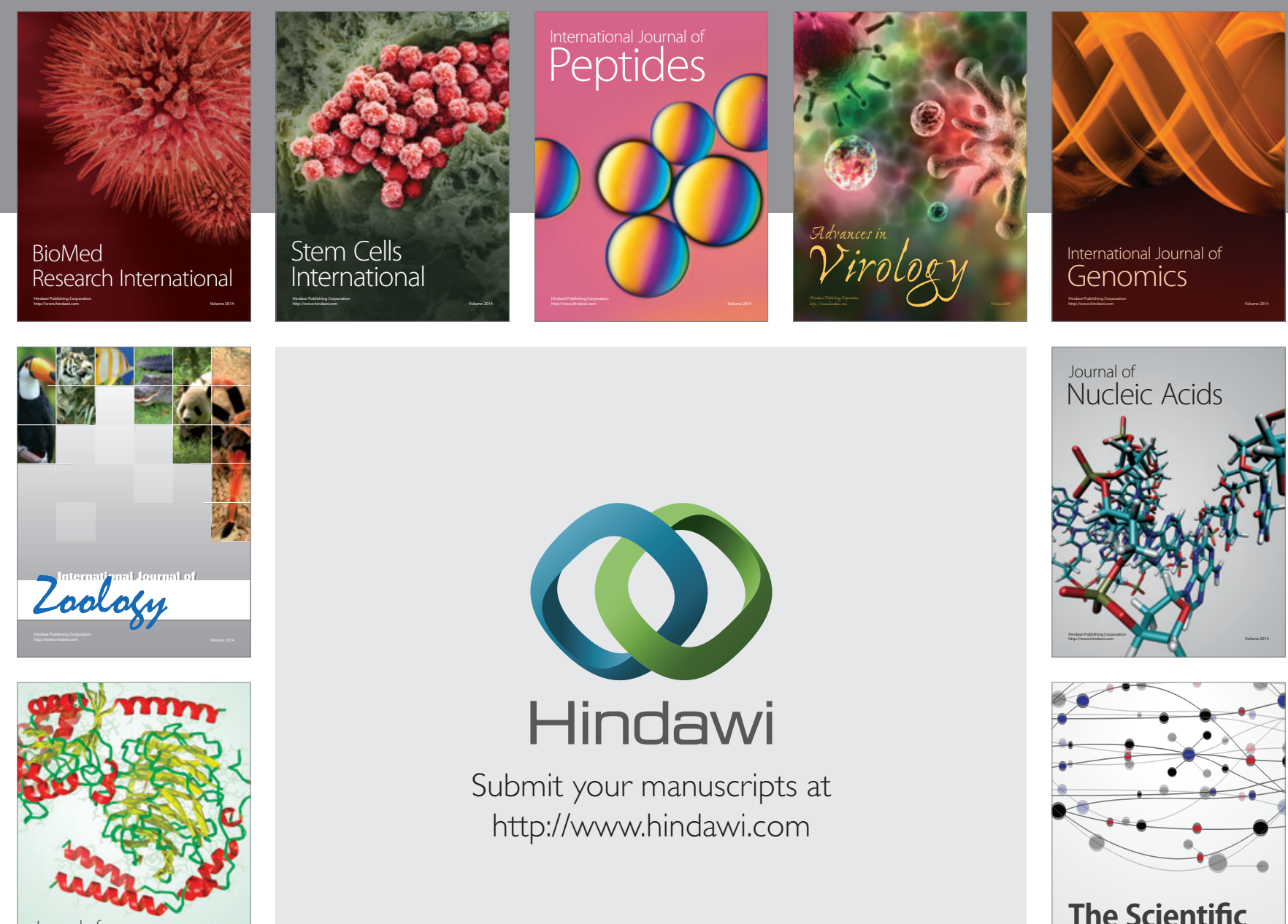

Submit your manuscripts at

http://www.hindawi.com

Journal of
Signal Transduction
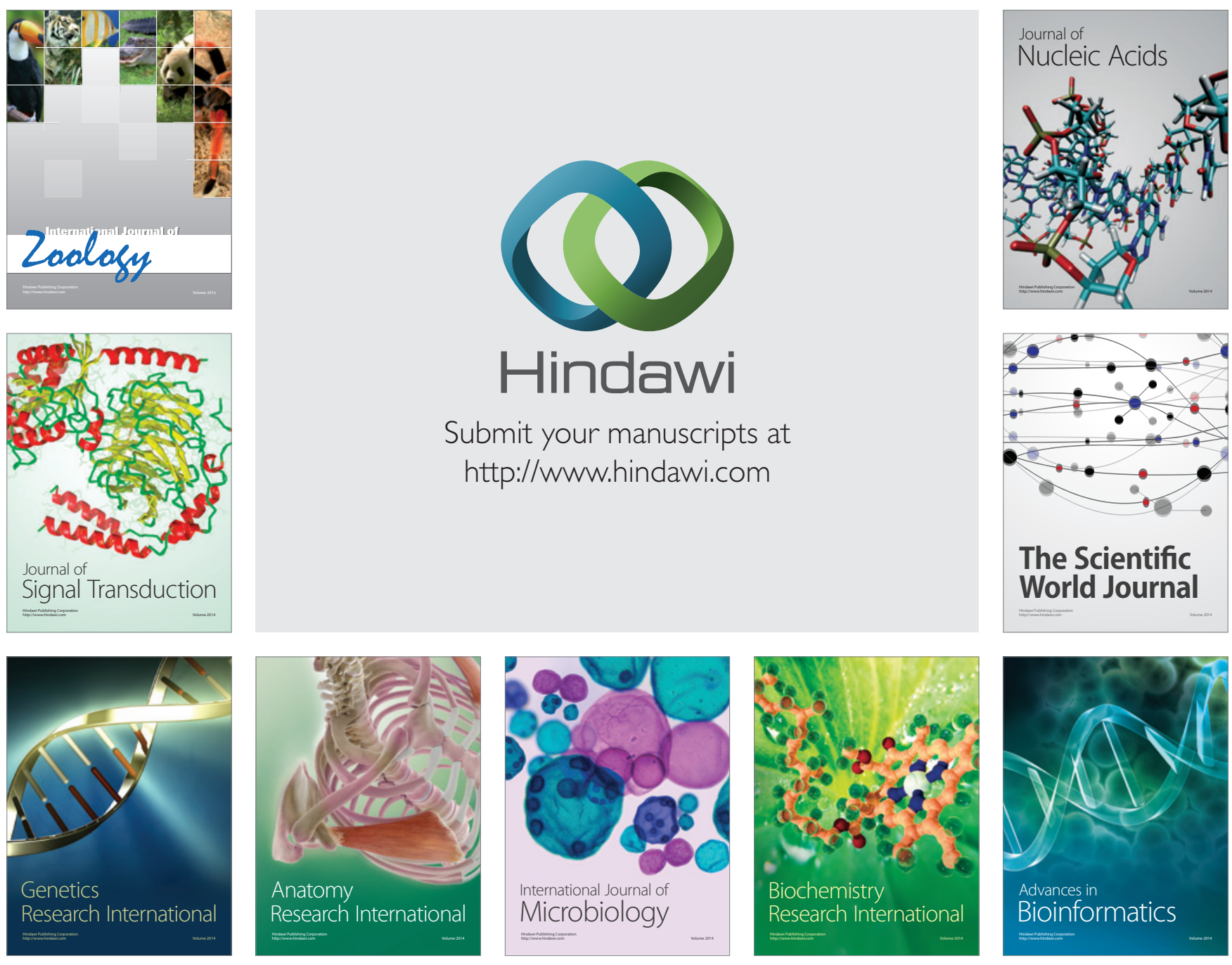

The Scientific World Journal
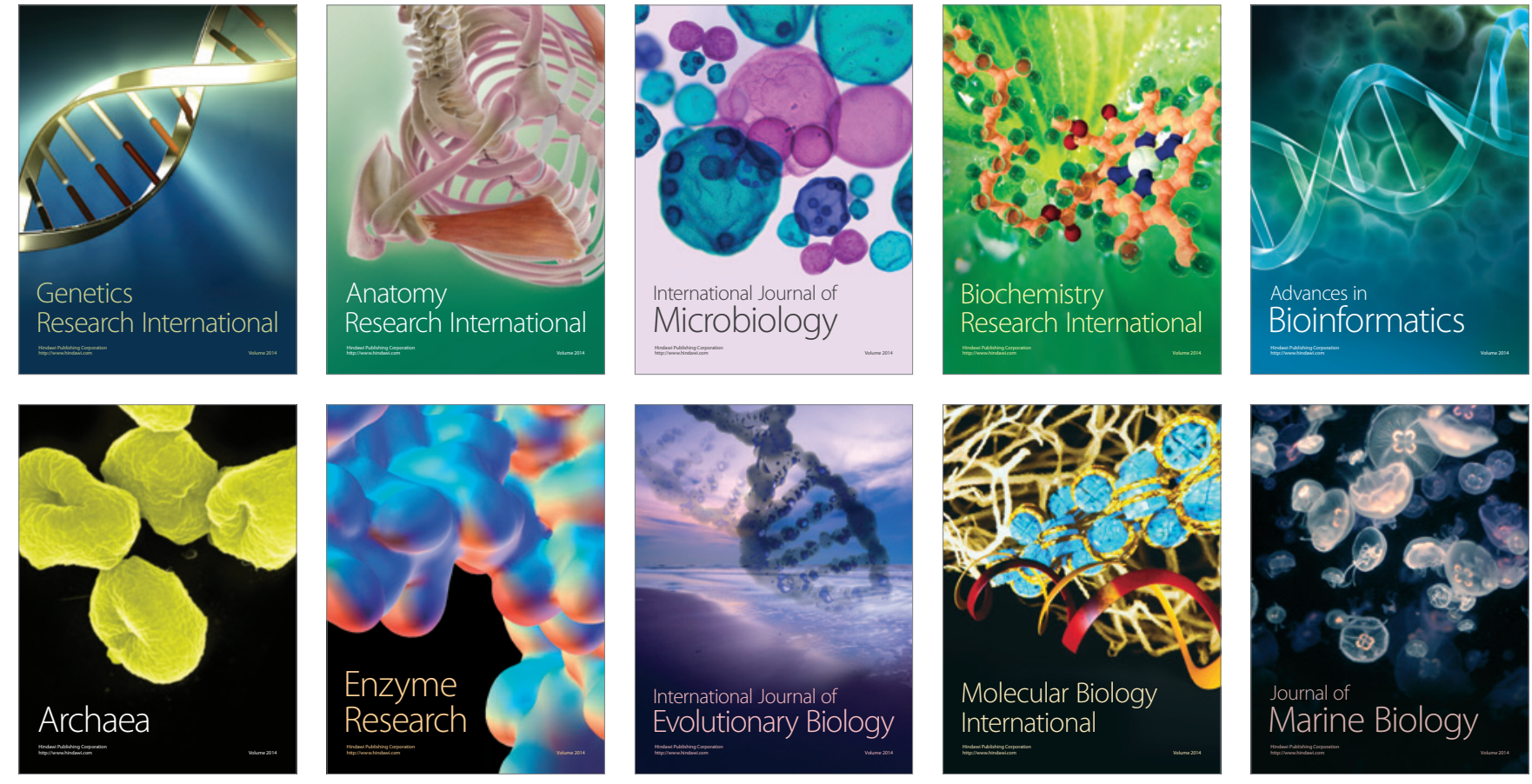